\title{
Growth, Electrical, Thermal, Optical, Physiochemical and NLO Properties of Dyes Doped Tetrafluoro Phthalate Crystals
}

\author{
B. LATHA ${ }^{1,2}$, P.KUMARESAN $^{2,3 *}$, S. NITHIYANANTHAM ${ }^{4 *}$ and K. SAMPATH KUMAR ${ }^{5}$ \\ ${ }^{1}$ Research \& Development Centre, Bharathiar University, Coimbatore, \\ Tamilnadu- 641048, India \\ ${ }^{2}$ Department of Physics, Rajalakshmi Engineering College, Thandalum, Chennai, \\ Tamilnadu- 602105, India \\ ${ }^{3}$ PG \& Research Department of Physics, Thiru.A.Govindasamy Government Arts College, \\ Tindivanam, Tamilnadu- 604002, India
}

${ }^{4}$ P.G \& Research Department of Physics, Thiru.Vi.Ka.Govt. Arts College, Thiruvarur, Tamilnadu- 610003, India

${ }^{5}$ PG\&Research Department of Physics, Arignar Anna Government Arts College, Villupuram, Tamilnadu- 605602, India

s_nithu59@rediffmail.com; lokeshkumaresan@yahoo.com

Received 16 July 2017 / Accepted 21 August 2017

\begin{abstract}
A systematic study has been carried out on the growth of pure and doped tetrafluoro phthalate crystals. The lattice parameters find through powder X-ray diffraction analysis for the grown pure and doped crystals. The functional groups, stretching are found through FT-IR and FT-Raman spectroscopical analysis, optical, absorption studies through UV-Vis analysis, thermal studies under TG/DTA analysis, through Vickers test and LCR metre the microhardness and dielectric studies were also carried out for the grown pure and dyes doped crystals. The SHG efficiency of the pure and dyes doped TFP crystals were also studied using Nd:YAG Q-switched laser. The results of these investigations are discussed in this paper.
\end{abstract}

Keywords: Tetrafluoro phthalate, Dyes, X-Ray diffraction, FT-IR, Hardness studies, SHG measurements

\section{Introduction}

In recent years, there has been considerable interest on organic materials due to their potential applications such as frequency conversion, optical signal processing, light modulation, optical switching and logic gates ${ }^{1-3}$. Semi-organic crystals are suitable for highly aligned and stable orientation of NLO chromophores in the crystal lattice ${ }^{4}$. In case of organic materials contain donor and acceptor groups positioned at either end of a suitable conjugation path. 
But, the organic materials having properties like optical damage threshold, wider transparency, necessary double refraction for phase matching with very high NLO coefficient, it helps for doubling the frequency ${ }^{5}$. Large crystals are needed for certain types of device applications $^{6-8}$. In recent times growing crystals of many organic, inorganic with neutral properties for non-linear optical (NLO) materials. The grown crystal should possess some physical properties like transparency, laser damage threshold and stable with varying temperature ${ }^{9-11}$.

Since, for the past three decades, one could see that there are lot of developments in science and technology-especially, in the fields of electronics, fibre-optic communication and lasers ${ }^{12}$. The scientific community achieving this development in functional materials through the single crystals of $\mathrm{Si}, \mathrm{Ge}$ and GaAs with NLO properties ${ }^{13}$. To enable a material to be potentially useful for NLO applications the material should be available in bulk tetrafluoro phthalate crystals (TFP). Also research into the growth of large single crystals TFP of from aqueous solution is currently serving as the important avenue to general progress in understanding many fundamental concepts of crystallization ${ }^{14}$. The effect of seed of different orientations on the habit of $\mathrm{c}$ and the change of morphology while doping with dyes has been investigated ${ }^{15}$.

\section{Experimental}

The TFP $\left(\mathrm{C}_{6} \mathrm{H}_{2} \mathrm{~F}_{4} \mathrm{O}_{4}\right)$ in Millipore water, the saturated solution TFP was prepared. The prepared solution was kept in the dust free atmosphere used clean-room. The prepared saturated solution of TFP was carefully filtered and poured in to the beaker and controls the nucleation near the surface region. The possible morphology of the TFP crystal of commonly growth face (001). TFP crystal cleavage faces find application as substrates in epitaxial techniques ${ }^{16}$. The TFP crystal was cleaving perpendicular to the (001) direction, this face and plane of crystal in this study ${ }^{17}$.

Under optimized condition highly transparent crystal growth was seen. The seed crystal was carefully placed at the bottom of beaker. The same procedure was repeated for dyes $(0.1$ mol\% methyl red, 0.1 mol\% rhodamine-B) doped TFP. Here, the infrared lamp replaced instead of programmable controller heater and the setup is shown in Figure 1.

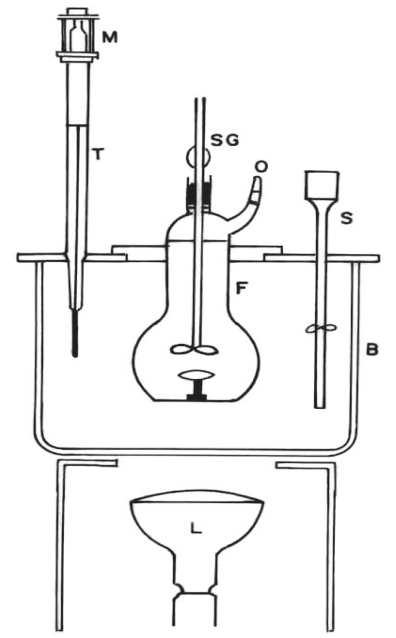
L: Heater lamp
B: Both
F: Flask
S: Stirrer
O: Opening
SG: Stirring Gland
T: Control Mometer
M: Motor

Figure 1. Schematic diagram of the growth apparatus 
The solutions were continuously agitated for $6 \mathrm{~h}$ at room temperature, filtered and kept free from vibration. Transparent dyes doped TFP crystals were obtained after period of 20 days. The photographs of as grown pure, methyl red and rhodamine-B doped TFP crystals (Figure 2a-c).

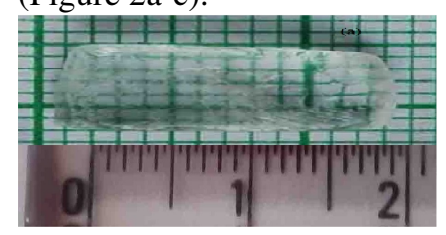

(a)

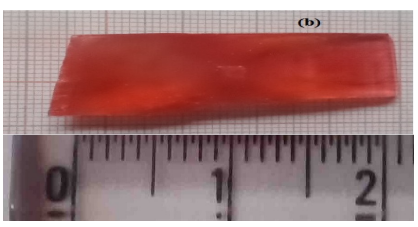

(a)

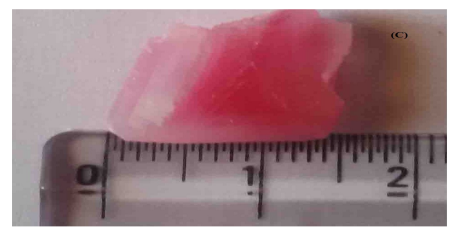

(a)

Figure 2. Grown crystals of (a) pure, (b) methyl red and (c) rhodamine-B doped TFP Crystals

\section{Characterisation}

The structural and lattice parameters are found through x-ray diffraction technique. The optical and absorption studies carried out through UV-Visible spectrometer. The compounds stretching and their functional groups and identified through Fourier transform infrared spectrometer. The hardness and the non-linear optical analysis with Vickers hardness and SHG set up. The dielectric and thermal properties are investigated through LCR meter and the TG/DTA techniques.

\section{Results and Discussion \\ XRD analysis}

The powder XRD patterns of pure and dyes TFP samples were refined using check cell software (Figure 3). The crystal parameters and the group are in good agreement with standard reference. No new peaks or phases were observed by doping with dyes. The peaks at (110) and (120) present in the pure TFP crystal and it are suppressed in doped TFP crystals. These peaks are not indexed as they are not matching with JCPDS data of pure TFP crystals. Similar peaks are observed by other reports as well. The system is found to exhibit monoclinic structure with the space group $\mathrm{P}_{21}$ and the lattice parameters of pure TFP crystal are $a=9.521 \AA, b=10.250$ $\AA$ and $\mathrm{c}=4.821 \AA$ which are in good agreement with the literature ${ }^{18}$. The pure and doped TFP crystals were cleaved into small pieces. The radiation used was MoK $\alpha$, monochromatized by graphite. Cell parameters were measured from 20 reflections and after refining the orientation matrix and cell parameters by least squares. Some additional peaks observed due to the existence of impurity and it affects reasonable optical properties. Due to change in morphology, slight change in intensity of some peaks were observed as a result of doping while the intensity of some peaks gets reduced as a result of doping ${ }^{19,20}$.

\section{UV-Vis spectral studies}

The recorded UV-Visible spectrum of ranges from 200-2000 nm are shown in Figure 4. The optical property oriented device having more transmittance range and transparency cut-off wavelength $^{21}$. A TFP crystal with the thickness of $2 \mathrm{~mm}$ without polishing and antireflection coating is used for the measurement. The lower cut off wavelength of TFP is around $300 \mathrm{~nm}$ and it has sufficient transmittance in the entire visible and NIR regions. It may be noted that the TFP crystal may possess the substantial contribution of the phonon subsystem ${ }^{22}$. It is good for optical window applications, because of no absorption in the region from $300 \mathrm{~nm}$ to $1100 \mathrm{~nm}$. The UV spectrum consists of far or vacuum ultraviolet region, near or quartz ultraviolet region and visible region. The region between $200-380 \mathrm{~nm}$ is called quartz ultraviolet region, normally 
termed as ultraviolet region. The region or spectral range most accessible for the instruments, is from 200 to $800 \mathrm{~nm}$ includes the visible region lying between $380-780 \mathrm{~nm}$ and region between $10-200 \mathrm{~nm}$ is vacuum ultraviolet region.

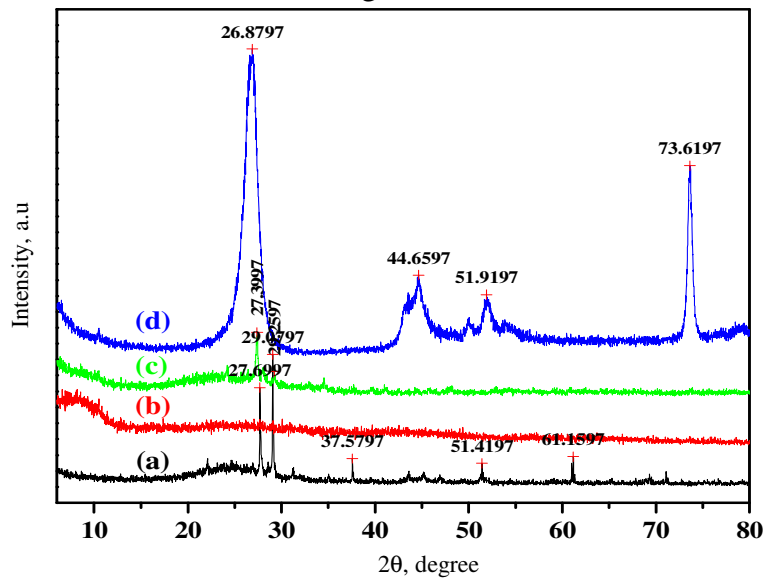

Figure 3. XRD-pattern for (a) pure TFP crystal (b) 0.1 mol \% rhodamine-B + TFP crystal, (c) 0.1 mol \% methyl red + TFP crystal and (d) 0.2 mol \% methyl red + TFP crystal

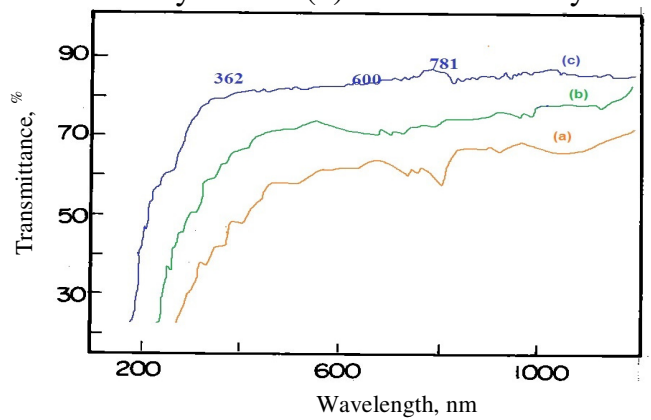

Figure 4. Transmittance spectra of (a) pure TFP (b) rhodamine-B doped TFP and (c) methyl red doped TFP crystal

From the spectra it is seen that the pure and dyes doped crystals have lower cut off wavelength of $193 \mathrm{~nm}$ and $254 \mathrm{~nm}$, but the pure crystal having wide output transmission from $243 \mathrm{~nm}$ to $1676 \mathrm{~nm}$. The presence of low cut-off wavelength and the wide optical transmission window are the desired properties for NLO activity. Moreover the doped crystals have reduced absorption and better transmission window of $254 \mathrm{~nm}-1672 \mathrm{~nm}$.

\section{FT-IR spectral analysis}

The FT-IR spectrum was recorded between $1000 \mathrm{~cm}^{-1}$ and $4000 \mathrm{~cm}^{-1}$, using BRUKER IFS 66V FTIR spectrometer. The FTIR spectra of pure and doped TFP appear almost similar to each other (Figure 5). C-H stretching frequencies of dye group are found between $3672 \mathrm{~cm}^{-1}$ and $3027 \mathrm{~cm}^{-1}$ for both pure and doped crystals. The characteristic peaks observed for zwitterions of dyes were present in the FTIR spectra of TFP. The peak at $2419 \mathrm{~cm}^{-1}$ for O-H stretching of carboxylic group. The $\mathrm{C}-\mathrm{H}$ stretching group is observed as a weak absorption at $3394 \mathrm{~cm}^{-1}$. The peaks at $1126 \mathrm{~cm}^{-1}, 1376 \mathrm{~cm}^{-1}$ and $1450 \mathrm{~cm}^{-1}$ are for the $\mathrm{C}=\mathrm{O}$ stretching of carboxylic group. 
Both the pure and doped compounds show absorption at $1636 \mathrm{~cm}^{-1}$ indicating the presence of primary amino group. The characteristic absorption for the -NH group in the aromatic ring is observed at $1944 \mathrm{~cm}^{-1}$ for TFP. The broad absorption around $3027 \mathrm{~cm}^{-1}$ indicates the co presence of $\mathrm{C}=\mathrm{O}$ stretching and $\mathrm{C}-\mathrm{H}$ stretching. Doping of metal ion into the crystal lattices does not show any significance change in absorption pattern.

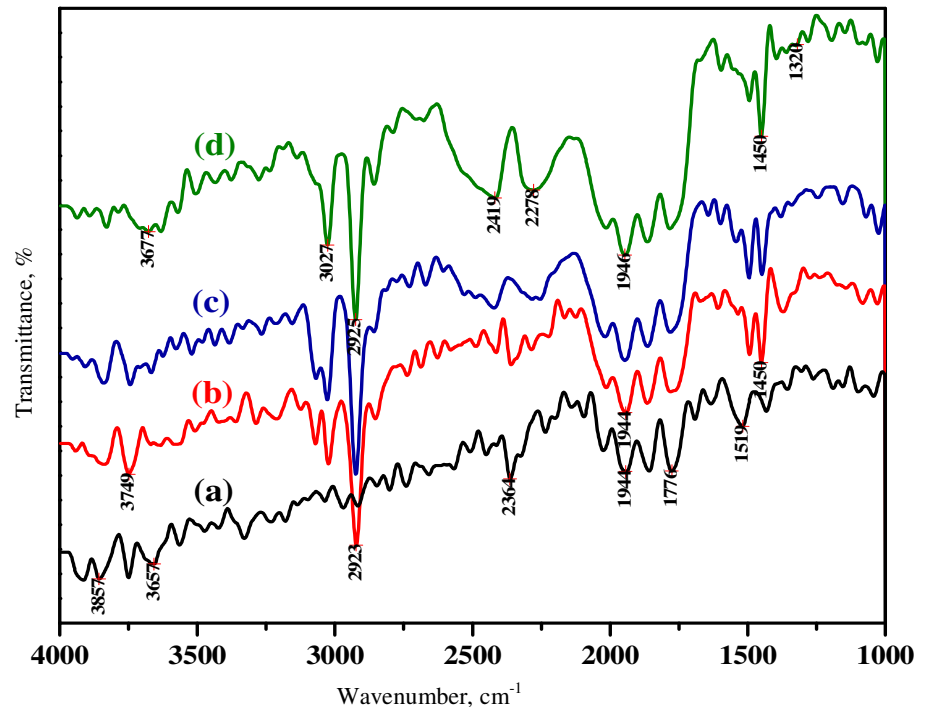

Figure 5. FTIR spectra of (a) pure TFP crystal (b) rhodamine-B doped TFP crystal (c) 0.1 mol \% methyl red doped TFP crystal and (d) 0.2 mol\% methyl red doped TFP crystal

Some of the absorptions show a marked difference in percentage transmittance. The percentage change of transmittance especially, at $824 \mathrm{~cm}^{-1}$ and $618 \mathrm{~cm}^{-1}$ are worth noting, because of the possibility of metal ions in intestinal place leads weak linkages. The TFP doping with rhodamine-B causes much broadening of the peaks in the spectra compared to the pure crystal. The spectra also reveal reduction in the crystal hardness as a result of dopant incorporation - this has been evident from the hardness measurements for pure and doped crystals. It is inferred that the modes due to polar groupings, for example $\mathrm{COOH}$, $\mathrm{COO}$ are more broadened compared to the modes of non-polar groupings illustrating that the doped crystals reject more degree of interaction for the polar groupings rather than for nonpolar groupings $\mathrm{s}^{23}$.

\section{Hardness studies}

Vickers hardness method is the reliable among the various methods of hardness measurement. The Vickers pyramid indenter whose opposite faces contained an angle $\left(\alpha=130^{\circ}\right)$ is the most widely accepted pyramid indenter. In general Hardness relates with the applied load and the indentation on surface area. The Vickers hardness number is thus calculated using the relation

$$
\mathrm{H}_{\mathrm{v}}=1.8544 \mathrm{p} / \mathrm{d}^{2} \mathrm{~kg} \mathrm{~mm}^{-2}
$$

Where $\mathrm{p}$ is the applied load in $\mathrm{kg}$ and $\mathrm{d}$ is the diagonal length of the indentation mark in $\mathrm{mm}$. Hardness values obtained through the size of impression after removal of the indenter. The mechanical behaviour of the pure and dyes doped TFP single crystals was analysed at room temperature. The chosen surface of the grown crystals were polished, washed and dried. Hardness measurements were taken for the applied load varying from 25 to $100 \mathrm{~g}$ 
keeping the indentation constant at $15 \mathrm{~s}$ for all the cases. It was found that both pure and doped TFP crystals are mechanically stable up to $100 \mathrm{~g}$. For loads above $100 \mathrm{~g}$ cracks started developing around the indentation mark which may be due to the release of internal stresses. The presence of cracks confirms the decrease in micro hardness. It was observed that amino acid added TFP crystals are highly stable towards the application of mechanical stresses. The observed changes in the micro hardness values of dyes doped TFP crystals may be due to the strong interaction of O-H groups of TFP with the COO groups of dyes ${ }^{24-26}$. Dyes doping in TFP improves the mechanical strength of microhardness value of pure and dye doped TFP were tabulated in Table 1 .

Table 1. Microhardness values of dyes doped TFP crystals

\begin{tabular}{ccc}
\hline S.No & Crystal & Microhardness $/ \mathrm{kg} \mathrm{mm}^{-2}$ ) \\
\hline 1 & TFP crystal & 139.79 \\
2 & Rhodamine-B doped TFP crystal & 145.56 \\
3 & Methyl red doped TFP crystal & 176.42 \\
\hline
\end{tabular}

\section{Thermal studies}

Figure 6 illustrates the differential thermal analysis (DTA) and thermogravimetric analysis (TGA) curves for the grown dye doped rhodamine-B crystal. The DTA curve shows that the material experiences an irreversible endothermic transition at $201.6{ }^{\circ} \mathrm{C}$, the temperature at which starts melting. The endothermic peak at which the substance starts melting which corresponds to the melting point at $210{ }^{\circ} \mathrm{C}$. The point of the trace corresponds to a vertical line. The better crystallinity of the crystal is due to the sharp endothermic peak. The exothermic peak at $280{ }^{\circ} \mathrm{C}$ indicates a phase change from liquid to vapour state as evident from the loss of weight of about $86 \%$ in TG curve.

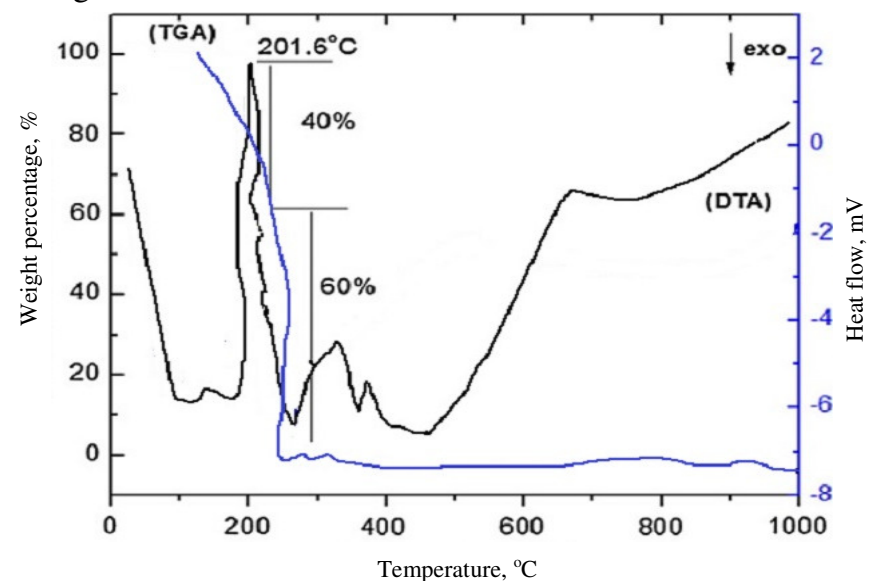

Figure 6. TG and DTA curves of the rhodamine-B doped TFP crystal

Thermal characteristics of the material were investigated by TG and DTA analysis. For the TG analysis (Figure 7), heated in the suitable air atmosphere. Figure 8 illustrates the TG and DTA curves for the grown methyl red doped TFP sample. The TG curve shows very small $(3.2 \%)$ weight loss up to $166^{\circ} \mathrm{C}$. Hence the material is thermally stable up to $127^{\circ} \mathrm{C}$ and above this temperature the material loses its weight gradually. As can be seen from the DTA curve in the figure, the material undergoes an endothermic transition at $67.3{ }^{\circ} \mathrm{C}$ where the melting begins. 


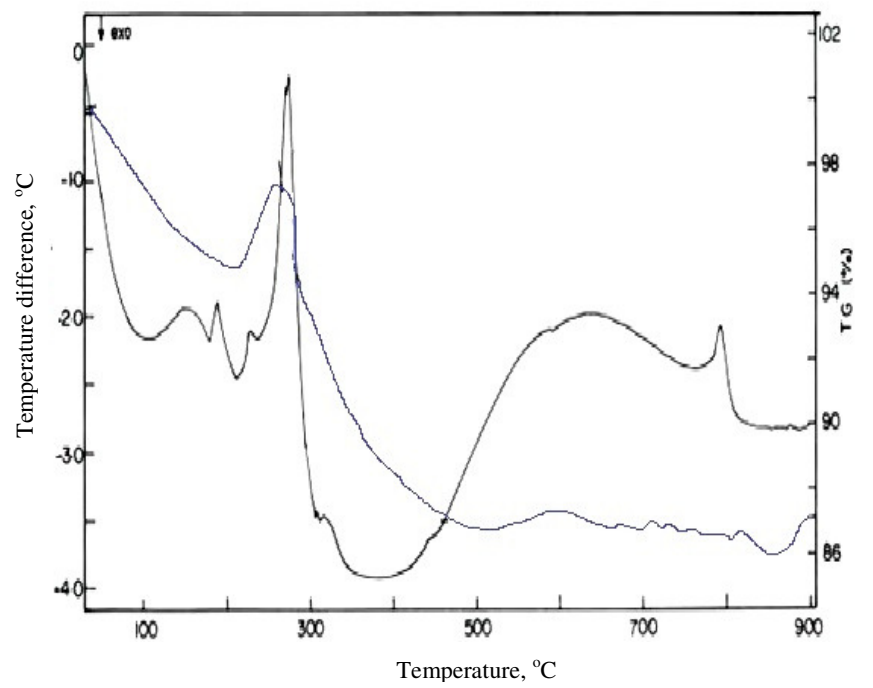

Figure 7. TG and DTA curves of the methyl red doped TFP crystal

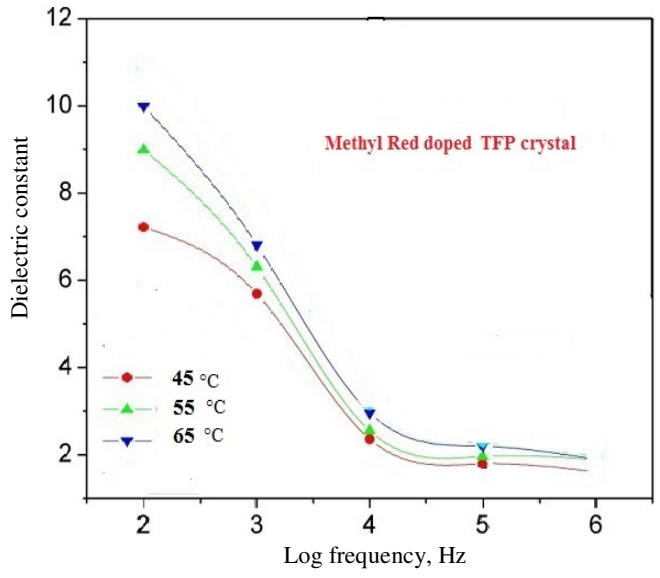

Figure 8. Dielectric studies on methyl red doped TFP crystal

The TG curve shows no weight loss during the first two endothermic peaks which confirms the phase transition of the material from solid to liquid (melting) and the weight of the material starts to losses after $128{ }^{\circ} \mathrm{C}$ is not due to self-degradation of TFP but merely its evaporation after melting. The peak at $235.23{ }^{\circ} \mathrm{C}$ indicates liquid phase to vapour phase as evident from the loss of weight in TG curve.

\section{Dielectric studies}

The temperature dependent dielectric constant of methyl red doped TFP crystals were measured using LCR Hi-Tester in the frequency range from $150 \mathrm{~Hz}$ to $100 \mathrm{MHz}$ at different temperatures. The electrical contact made by silver coating with electrodes set up ${ }^{27}$. Here, the crystals are acted as a dielectric medium and the capacitor plates acts as electrodes. The variation of capacitance was recorded in the frequency range $150 \mathrm{~Hz}$ to $100 \mathrm{MHz}$ at different temperatures. 
The dielectric constant of the methyl red (dye) doped TFP was calculated for different measured frequencies from the capacitance values. It is observed that the dielectric constant values having negative trend with applied frequency. i.e., high $\varepsilon_{\mathrm{r}}$ at low region and decreases the applied increasing frequency (Figure 8). The very high value of dielectric constant at low frequencies may be due to the presence of all the four polarizations namely, space charge, orientation, electronic and ionic polarization ${ }^{28}$ and the low value at higher frequencies may be due to the loss of significance of these polarizations gradually.

The most commonly measured small signal electrical property is the dielectric permittivity $\varepsilon_{\mathrm{r}}$ also often referred as the dielectric constant. $\varepsilon_{\mathrm{r}}$ in polar materials is rarely a constant, but varies with the applied field, stress and temperature and other parameters. When a dielectric material is subjected to an electric field, the net polarization of the medium is altered. The dielectric constant and dielectric loss may be obtained from a measurement of the real and complex admittance of a crystal. These measurements are particularly straightforward with impedance bridges, which separate the conductance and capacitance of the crystals. Pure crystals have been used for the measurement and this is mainly used for the identification of phase transitions and the recording of transition temperature. The in homogeneities in the crystal due to strain leads to broaden the phase transition cause no apparent discontinuity in $\varepsilon_{\mathrm{r}}$.

\section{Second harmonic generation efficiency test}

The fundamental of an Nd:YAG laser $(1064 \mathrm{~nm})$ can be converted to $532 \mathrm{~nm}$ of second harmonic or its $355 \mathrm{~nm}$ of third harmonic or its $266 \mathrm{~nm}$ of fourth harmonic by using TFP crystals. It is necessary to analyse the performance in frequency conversion of the grown crystals $^{29}$. And it is important to find and analyse harmful absorption bands in non-linear optical crystals. In order to confirm the suitability of the methyl red doped TFP crystal and rhodamine-B doped TFP crystal and the harmonic generation was tested using the Nd-YAG laser. A tiny crystal was mounted on the sample holder and subjected to YAG laser allow to pass through the crystal, the output of green light from the input light, the SHG was analysed and all the values are shown in Table 2.

Table 2. Comparison of SHG of pure and dyes doped TFP crystals

\begin{tabular}{ccccc}
\hline $\begin{array}{c}\text { S. } \\
\text { No. }\end{array}$ & Sample & $\begin{array}{c}\text { Input power } \\
/ \mathrm{mJ}\end{array}$ & $\begin{array}{c}\text { Output } \\
\text { power / mJ }\end{array}$ & $\begin{array}{c}\text { SHG efficiency } \\
\text { relative to KDP }\end{array}$ \\
\hline 1 & TFP Crystal & 6.5 & 103.3 & 1.95 \\
\hline 2 & $\begin{array}{c}\text { Rhodamine-B doped } \\
\text { TFP crystal }\end{array}$ & 6.5 & 117.1 & 2.25 \\
\hline 3 & $\begin{array}{c}\text { Methyl red doped TFP } \\
\text { crystal }\end{array}$ & 6.5 & 129.8 & 2.45 \\
\hline
\end{tabular}

The efficiency of dye doped methyl red doped TFP crystals were compared with pure TFP and also show that methyl red doped TFP crystal has higher efficiency. A sample of KDP was used for the same experiment as a reference material in the SHG measurement. It was found that the frequency doubling efficiency of the doped TFP was better than KDP. It is already established that the molecular hyperpolarizability and mechanical stabilities get enhanced in organic molecules containing $\mathrm{OH}$ and $\mathrm{NH}$ groups, which possess in hydrogen bond interactions. The estimated SHG efficiencies of pure TFP, rhodamine-B and methyl red doped TFP crystals were found to be 1.95, 2.25 and 2.45 times greater than that of KDP crystal respectively. Hence methyl red doped TFP crystal exhibit better NLO character than 
pure and rhodamine -B dye doped TFP crystal $^{30}$. The results confirm the suitability of dyes doped TFP crystals in NLO application.

\section{Conclusion}

The pure and dyes doped TFP crystals were grown by slow evaporation method. The crystalline parameters and monoclinic structure were confirmed by single crystal x-ray diffraction analysis. Functional groups present in the grown crystal were confirmed by FTIR spectral studies. The presence of functional groups was identified by FTIR analysis. There results suggest that the doped specimens are suitable prime materials in optoelectronic device applications.

The optical behaviour has been evaluated by UV-Visible studies and it substantiates the suitability for optoelectronic applications. The optical characters of the samples were assessed by UV-Visible studies. Dyes doped TFP crystals show a better transmission value of 76\% (Methyl red doped TFP) and 67\% (Rhodamine-B doped TFP) than the pure crystal. Thermal studies revealed that TFP is thermally stable up to $185^{\circ} \mathrm{C}$. Mechanical properties of the title materials were evaluated by Vickers micro hardness test and it confirms that both the pure and doped materials belong to soft material category. The dielectric constant and dielectric loss studies of TFP establish the normal behaviour. The double refraction and dielectric constant measurements confirmed the nonlinear optical applications.

In order to understand the behaviour of any solid material, the structural characterization is carried out first. The optical studies suitable for transparency window and cut- off frequency of the crystal. Single crystals grown by the various methods need to be characterized to assess the suitability of the crystal for various applications including NLO device application. Since the entire thrust is with respect to NLO application, the SHG property is estimated. NLO studies confirm that methyl red doped TFP crystals are best suited than dyes doped TFP crystals for NLO device applications possessing SHG efficiency. The existence of phase matching property, which concludes dyes added TFP crystals are eligible candidates in frequency doubling applications.

\section{Acknowledgement}

The authors gratefully acknowledge financial supports from the Department of Science and Technology-Science and Engineering Research Board [DST-SERB-FAST TRACK, PS20/2009(SR)], New Delhi, India.

\section{References}

1. Ramajothi J and Dhanuskodi S, Cryst Res Technol., 2003, 38, 986-991; DOI:10.1002/crat.200310125

2. Nakamoto K, IR Spectra of Inorganic and Coordination Compounds, Second Ed., Wiley, New York, 1978.

3. Akkurt M, Ozturk S, Ramajothi J, Buyukgungor $\mathrm{O}$ and Dhanuskodi S, Acta Crystallogr., 2004, E60, 481-483; DOI:10.1107/S1600536804004180

4. Silverstein R M, Basseler G C and Morill T C, Spectrometric Identification of Organic Compounds, fifth Ed., Wiley, New Delhi, 1998.

5. Ramajothi J and Dhanuskodi S, J Crystal Growth, 2006, 289, 217-223; DOI:10.1016/j.jcrysgro.2005.10.103

6. Xun Feng, Rong Fang Li, Yang Wang and Pan Hao Tian, New Crystal Structure, 2016, 4, 1139-1141; DOI:10.1515/ncrs-2016-0092

7. Sivakumar B, Gokul Raj S, Ramesh Kumar G and Mohan R, Bull Korean Chem Soc., 2012, 33(11), 3755-3760; DOI:10.5012/bkcs.2012.33.11.3755 
8. Sivakumar B, Gokul Raj S, Ramesh Kumar G and Mohan R, J Crystal Proc Technol., 2012, 2(4), 130-136; DOI:10.4236/jcpt.2012.24018

9. Loring J S, Karlsson M, Faweett W R and Casey W H, Spectrochimica Acta Part A, 2001, 57(8), 1635-1642; DOI:10.1016/S1386-1425(01)00391-2

10. Kejalakshmy N and Srinivasan K, Opt Mater., 2004, 27, 389-394; DOI:10.1016/j.optmat.2004.09.005

11. Kumari L and Li W Z, Cryst Res Technol., 2010, 45, 311-315; DOI:10.1002/crat.200900600

12. Senthil A and Ramasamy P, J Cryst Growth, 2009, 311(23-24), 4720-4724; DOI:10.1016/j.jcrysgro.2009.09.014

13. Bruice P Y, Organic Chemistry, Third Ed., Pearson Education Asia, Singapore, 2002.

14. Sajan D, Vijayan N, Safakath K, Philip R and Joe I H, J Phys Chem A, 2011, 115, 8216-8226; DOI:10.1021/jp201818y

15. E. Samsoe, N. Kjaergaard, H. Lausen, P.E. Andersen, P.M. Petersen, Opt. Comm.245 (2005) pp.333-339. doi.org/10.1016/j.optcom.2004.10.023

16. Kuppers H, Takusagawa F and Koetzle T F, J Chem Phys, 1985, 82(12), 5636-5647; DOI:10.1063/1.448550

17. Sangwal K and Zaniewska G, J Mater Sci., 1984, 19(4), 1131-1144; DOI:10.1007/BF01120022

18. Satapathy S, Sharma S K, Karnal A K and Wadhawan V K, J Cryst Growth, 2002, 240, 196-202; DOI:10.1016/S0022-0248(01)02135-2

19. Meenakshisundaram S, Parthiban S, Pisipaty U R, Madhurambal G and Mojumdar S C, J Therm Anal Calorim., 2010, 100, 821-826; DOI:10.1007/s10973-010-0738-4

20. Sankaranarayanan K and Ramasamy P, J Cryst Growth, 2005, 280, 467-473; DOI:10.1016/j.jcrysgro.2005.03.075

21. Kejalakshmy N and Srinivasan K, J Phys D, 2003, 36(15), 1778-1782.

22 Sander Graswinckel W, Fieke J. Van den Bruele, Willem J P Van Enckevort and Elias Vlieg, Cryst Growth Des., 2007, 7, 243-249; DOI:10.1021/cg0602257

23. Bredas J L, Adant C, Tackx P and Persoons A, Chem Rev., 1994, 94(1), 243-278; DOI:10.1021/cr00025a008

24. Laud B B, Lasers and Non Linear Optics, New Age International Publishers, New Delhi, Inida, $3^{\text {rd }}$ Edition, 2011.

25 Thilagavathy S R, Rajesh P, Ramasamy P and Ambujam K, Spectrochimica Acta Part A: Mole Biomole Spectro., 2014, 127, 248-255; DOI:10.1016/j.saa.2014.01.137 1386$1425 / 2014$

26. Krishnan S, Justin Raj C, Dinakaran S, Uthrakumar R, Robert R and Das S J, J Phys Chem Solids, 2008, 69, 2883-2887; DOI:10.1016/j.jpcs.2008.06.146

27. Senthil A, Ramasamy P and Bhagavannarayana G, J Crystal Growth, 2009, 311(9), 2696-2701; DOI:10.1016/j.jcrysgro.2009.02.036

28. Miniewicz A, Lefebvre J, Fontaine H and Marqueton Y, J Raman Spectr., 1990, 21, 177-183; DOI:10.1002/jrs.1250210305

29. Balamurugan N, Lenin M and Ramasamy P, Mater Lett., 2007, 61(8-9), 1896-1898; DOI:10.1016/j.matlet.2006.07.184

30. Rumi M and Perry J W, Adv Opt Photon, 2010, 2, 451-518;

DOI:10.1364/AOP.2.000451 